\title{
Obese Foveal Avascular Zone Assessed by Optical Coherence Tomography Angiography of the Retina: Is There a Relation to Comorbities?
}

\author{
Jamilla Viana Silva Asfora1, Márcio Bittar Nehemy², \\ Diego Nery Benevides Gadelha ${ }^{1}$, Carlos Teixeira Brandt ${ }^{{ }^{*}}$ \\ ${ }^{1}$ Medical School, UNIFACISA, Campina Grande, Paraíba, Brazil \\ ${ }^{2}$ Ophthalmology, Federal University of Minas Gerais, Belo Horizonte, Brazil \\ Email: *carlosbrandt@bol.com.br
}

How to cite this paper: Asfora, J.V.S. Nehemy, M.B., Gadelha, D.N.B. and Brandt, C.T. (2021) Obese Foveal Avascular Zone Assessed by Optical Coherence Tomography Angiography of the Retina: Is There a Relation to Comorbities? Open Journal of Ophthalmology, 11, 176-190. https://doi.org/10.4236/ojoph.2021.112014

Received: February 26, 2021

Accepted: May 28, 2021

Published: May 31, 2021

Copyright $\odot 2021$ by author(s) and Scientific Research Publishing Inc. This work is licensed under the Creative Commons Attribution International License (CC BY 4.0).

http://creativecommons.org/licenses/by/4.0/

\section{(c) (i) Open Access}

\begin{abstract}
Purpose: To investigate the foveal avascular zone (FAZ) in obese by optical coherence tomography angiography (OCT-A) and to evaluate the findings of structural optical coherence tomography (OCT) and their relations with comorbidities. Methods: It was included 35 obese (study group) and 30 normal individuals (control group). Patients with retinal diseases and retinal treatments were excluded. The images were obtained using the Topcon ${ }^{\circledR}$. Results: The mean areas of FAZ in superficial plexus (FAZ-SP) and deep plexus (FAZ-DP) were significantly greater in the study group: FAZ-SP was $405.0 \pm 136.4 \mu \mathrm{m}^{2}$ in the obese group and $307.3 \pm 78.6 \mu \mathrm{m}^{2}$ in the control group and in the left eye (LE) $477.1 \pm 124.4 \mu \mathrm{m}^{2}$ in the obese group and 384.0 $\pm 88.7 \mu \mathrm{m}^{2}$ in the control group. This difference was statistically significant (RE: $\mathrm{p}=0.0014$ and LE: $\mathrm{p}=0.0012$ ). The mean area of the FAZ-DP was 491.0 $\pm 124.4 \mu \mathrm{m}^{2}$ (Right eye-RE) in the obese group and $384.4 \pm 88.7 \mu \mathrm{m}^{2}$ in the control group and in the left eye (LE) was $497.9 \pm 124.1 \mu \mathrm{m}^{2}$ in the obese group and $484.9 \pm 92.7 \mu \mathrm{m}^{2}$ in the control group. There were no correlations regarding FAZ-SP and FAZ-DP in both eyes with fasting blood glucose, glycated hemoglobin, total cholesterol and fractions and triglycerides. A significant association between enlargement of FAZ-DP and type 2 diabetes mellitus $(\mathrm{p}=0.0160)$ was observed. Conclusion: The FAZ areas in superficial and deep plexus achieved significantly greater values in the study group. There was a significant association between a larger deep FAZ area and type 2 diabetes mellitus. It is necessary an evaluation with a larger sample size to corroborate the findings.
\end{abstract}

\section{Keywords}

Obesity, Retina, Fovea Centralis, Retinal Vessels, Optical Coherence 
Tomography Angiography

\section{Introduction}

Obesity is excessive fat accumulation, diagnosed by a body mass index (BMI) $\geq$ $30 \mathrm{~kg} / \mathrm{m}^{2}$ [1]. It has become a major public health problem at a pandemic level

[1] [2]. It is associated with an increase in risk factors for morbidities including diabetes, cardiovascular and kidney diseases, cancer and eye diseases [3]-[11]. In addition, obesity is associated with changes in lipid metabolism and hypoxia and low blood flow that can affect various tissues, particularly the retina, which is highly dependent on arterial flow and good oxygenation [12].

The retina presents high oxygen consumption, generating intense oxidative stress and its radicals, which makes it vulnerable over time. Modifications of the amounts of oxygen can alter its outer layer and the retinal pigment epithelium (RPE), creating a diverse gradient of oxidative damage in the photoreceptor components of the inner layer [12]. Additionally, the retinal photoreceptors contain high levels of polyunsaturated fatty acids. These peculiarities of the oxidative and lipid metabolism of the retina predispose to degenerative events. The protection for this continuous aggression can prevent its degeneration [13].

Although there are several studies about obesity, its forms of treatment and ophthalmological complications, there is still a lack of controlled studies in Brazil on this subject [14] and specifically on changes in the foveal avascular zone (FAZ), in which there may be a decrease retinal microvasculature and enlargement of this area due to vascular interruption [15]. It should be emphasized that non-invasive and safe monitoring of the FAZ dimensions can be an important approach for early diagnosis, monitoring and treatment of type II diabetes, arterial hypertension and possibly obesity with and without comorbidities [16] [17] [18] [19].

With the optical coherence tomography (OCT) and with high-resolution structural and vascular image of the entire thickness of the retina and choroid through optical coherence tomography angiography (OCT-A), there was an increase in the interest for this new tool to study changes in systemic medical conditions [16] [17] [18] [19].

The assessment of the hemodynamic status of the retina of obese individuals is made possible using OCT-A, allowing the measurement of FAZ and detecting early possible systemic vascular changes [18] [19]. As far as we know, there are no published studies on this topic, and the present study proposes to evaluate, through OCT-A, FAZ in obese individuals and the possible relations between FAZ and comorbidities, including parameters of clinical evaluation, biochemical tests and lifestyle habits.

\section{Methods}

The observational, cross-sectional and analytical study was performed at the Re- 
tina Department at the Hospital da Visão (Eye Care Center) in Campina Grande-Paraiba Brazil from February 2019 to February 2020.

Thirty-five obese individuals with or without comorbidities (study group) were included, according to the BMI classification (BMI greater than or equal to 30 ). In the control group, 30 individuals without obesity (BMI between 18.5 and 24.9) and without clinical or ophthalmologic comorbidities were recruited. The ages ranged from 26 to 58 years in the study group and from 20 to 57 years in the control group. Individuals were cared at the Hospital da Visão. Those who met the criteria were invited to voluntarily participate in the research and sent to the Retina Department.

Individuals with endogenous obesity (such as Cushing's syndrome) were excluded. Retinal diseases such as vascular occlusions, age-related macular degeneration (AMD), uveitis, retinal dystrophies, as well as patients with a history of intraocular surgery, macular photocoagulation (focal or grid), panphotocoagulation and intra-vitreous injections were also excluded.

All information from these individuals was collected: identification, anthropometric data, biochemical data and lifestyle.

Anthropometric data were measured: weight, height, BMI and waist circumference. Biochemical data from the last six months (fasting blood glucose, glycated hemoglobin, total cholesterol and fractions and triglycerides) were collected.

The patients were stratified according to the existing morbidities and underwent a complete eye exam. In addition, it was obtained OCT images: central macular thickness, subfoveal choroidal thickness, mean peripapillary retinal nerve fiber layer thickness, superior peripapillary retinal nerve fiber layer thickness, inferior peripapillary retinal nerve fiber layer thickness, nasal peripapillary retinal nerve fiber layer thickness and temporal peripapillary retinal nerve fiber layer thickness and OCT-A (superficial plexus and deep plexus). Best corrected visual acuity was measured using the Snellen table and was converted to LogMAR for statistical analysis.

The images of the OCT and OCT-A of $3 \times 3 \mathrm{~mm}$ were obtained using the Topcon ${ }^{\circledR}$ DRI OCT-1 Triton/Plus. All images were evaluated by two investigators with the measurement of the FAZ area performed manually. Three consecutive measurements were taken by each examiner and the mean of these measurements was expressed at the end.

The results of the quantitative variables were expressed by their means and standard deviations (SD). Qualitative variables were expressed by their absolute and relative frequencies. Student's t test was used to assess differences between means when the two numerical sets passed the normality test. If not, the Mann Whitney test was used. For comparison between means of more than two numerical sets, the ANOVA test was used, if the value was equal to or less than 0.05 , the Kruskal-Wallis test was used. Fisher's exact test and chi-square test were used to verify differences between frequencies. In order to assess the possi- 
ble correlation between clinical parameters and the FAZ areas, Pearson's linear correlation test was used when the two numerical sets passed the normality test and the Spearman correlation test if the sets did not pass the normality test. $\mathrm{p} \leq$ 0.05 was considered as the limit for rejecting the null hypothesis.

The project was approved by the UNIFACISA Human Research Ethics Committee. Patients were made aware of the details of the research and signed the Free and Informed Consent Form.

\section{Results}

Thirty-five obese were recruited, 19 (63.3\%) women and 11 (36.7\%) men. The ages in the study group ranged from 26 to 58 years, with a mean of $42.7 \pm 9.5$ years. In the control group, 30 individuals were recruited, 24 (80\%) women and $6(20 \%)$ men, with ages ranging from 20 to 57 years, with a mean of $37.6 \pm 11.0$ years. Thus, homogeneity was established in relation to age $(\mathrm{p}=0.0515)$ and frequency of male and female in both groups $(\mathrm{p}=0.2516)$.

Thirteen (37.1\%) of the obese had systemic arterial hypertension, $10(28.6 \%)$ were diabetic, 13 (37.1\%) had no comorbidities, 4 (11.4\%) were smokers and 9 (25.7\%) practiced regular physical activity. Regarding the classification of obesity: $20(57.1 \%)$ had class I obesity, $6(17.1 \%)$ were class II and 9 (25.7\%) were class III. The $35(100 \%)$ obese had abdominal obesity with abdominal circumference ranging from 90 to $150 \mathrm{~cm}$, with a mean of $109.5 \pm 11.6 \mathrm{~cm}$ and $12(34.3 \%)$ had metabolic syndrome. In the control group, none of the individuals had systemic arterial hypertension or diabetes, 3 (10\%) were smokers and $12(40 \%)$ practiced regular physical activity. None had abdominal obesity or met criteria for metabolic syndrome. The frequency of smoking $(\mathrm{p}=0.4716)$ and physical activity ( $p=0.5889$ ) were homogeneous between the two groups.

Weight, BMI (body mass index) and IOP (intraocular pressure) in both eyes had significantly higher measurements in the study group $(\mathrm{p}=0.0001, \mathrm{p}=$ $0.0001, p=0.0001$ in both eyes) while height measurements did not differ between the two groups $(\mathrm{p}=0.7644)$ (Table 1$)$.

The FAZ area, both the superficial plexus (FAZ-SP) and the deep plexus (FAZ-DP), achieved significantly higher values in the study group. The mean area of the FAZ-SP was $405.0 \pm 136.4 \mu \mathrm{m}^{2}$ in the right eye in the obese group and $307.3 \pm 78.6 \mu \mathrm{m}^{2}$ in the control group and in the left eye $477.1 \pm 124.4 \mu \mathrm{m}^{2}$ in the

Table 1. Clinical characteristics of the study and control groups.

\begin{tabular}{cccc}
\hline Parameters & Study $(\mathrm{n}=35)$ & Control $(\mathbf{n}=30)$ & p value \\
\hline Age (years) & $42.7 \pm 9.5$ & $37.6 \pm 11.0$ & 0.0515 \\
Weight $(\mathrm{kg})$ & $95.1 \pm 17.6$ & $62.0 \pm 8.1$ & 0.0001 \\
Height $(\mathrm{cm})$ & $162.4 \pm 10.1$ & $163.8 \pm 8.3$ & 0.5098 \\
BMI $\left(\mathrm{kg} / \mathrm{m}^{2}\right)$ & $35.9 \pm 4.9$ & $23.0 \pm 1.6$ & 0.0001 \\
& RE: $15.9 \pm 2.8$ & RE: $13.2 \pm 2.2$ & RE: 0.0001 \\
IOP $(\mathrm{mmHg})$ & LE: $16.2 \pm 2.9$ & LE: $13.2 \pm 2.1$ & LE: 0.0001 \\
\hline
\end{tabular}


obese group and $384 \pm 88.7 \mu \mathrm{m}^{2}$ in the control group. This difference was statistically significant (RE: $p=0.0014$ and LE: $p=0.0012$ ). The mean area of the FAZ-DP was $491.0 \pm 124.4 \mu \mathrm{m}^{2}$ in the right eye in the obese group and $384.4 \pm$ $88.7 \mu \mathrm{m}^{2}$ in the control group and in the left eye was $497.9 \pm 124.1 \mu \mathrm{m}^{2}$ in the obese group and $484.9 \pm 92.7 \mu \mathrm{m}^{2}$ in the control group. The differences between the two groups were also statistically significant $(\mathrm{p}=0.0012$ in the RE and $\mathrm{p}=$ 0.0005 in the LE).

No significant differences were found between the two groups for measures of central macula thickness (CMT: central macular thickness), subfoveal choroid thickness (SFChT: subfoveal choroidal thickness), mean peripapillary retinal nerve fiber layer thickness (M-RNFL), superior peripapillary retinal nerve fiber layer thickness (S-RNFL), inferior peripapillary retinal nerve fiber layer thickness (I-RNFL), nasal peripapillary retinal nerve fiber layer thickness (N-RNFL) and temporal peripapillary retinal nerve fiber layer thickness (T-RNFL).

Table 2 shows the means and standard deviations of the OCT-A and structural OCT measurements of the study and control groups.

The means of measurements made independently by two observers did not reveal a significant difference $(\mathrm{p}>0.05)$. Similarly, the correlation between measurements made by these observers revealed a strong correlation $(r=0.84)$ (Figure 1$)$.

In eyes of the control group, significantly smaller areas of FAZ-SP and FAZ-DP were observed, as shown in an OCT-A image in Figure 2, reflecting the hemodynamic pattern considered normal.

Table 2. Comparison between findings of structural OCT and OCT-A in the study and control groups.

\begin{tabular}{|c|c|c|c|}
\hline Measures & Obese & Control & $\mathrm{p}$ value \\
\hline \multirow{2}{*}{ CMT $(\mu \mathrm{m})$} & OD $229.8 \pm 16.7$ & OE $227.8 \pm 15.5$ & 0.8953 \\
\hline & OE $233.7 \pm 22.2$ & OE $225.0 \pm 17.8$ & 0.1995 \\
\hline \multirow{2}{*}{$\operatorname{SFChT}(\mu \mathrm{m})$} & OD $260.9 \pm 36.1$ & OD $264.0 \pm 44.9$ & 0.9843 \\
\hline & OE $261.4 \pm 38.0$ & OE $257.5 \pm 42.1$ & 0.6955 \\
\hline \multirow{2}{*}{ M-RNFL $(\mu \mathrm{m})$} & OD $106.8 \pm 16.7$ & OD $110.2 \pm 1.0$ & 0.8332 \\
\hline & OE $106.1 \pm 14.8$ & OE $110.5 \pm 10.7$ & 0.1763 \\
\hline \multirow{2}{*}{ S-RNFL $(\mu \mathrm{m})$} & RE $133.0 \pm 24.6$ & RE $134.5 \pm 17.9$ & 0.7671 \\
\hline & LE $132.8 \pm 24.9$ & LE $143.0 \pm 17.4$ & 0.0586 \\
\hline \multirow{2}{*}{ I-RNFL $(\mu \mathrm{m})$} & RE $139.6 \pm 25.2$ & $\operatorname{RE} 143.2 \pm 17.7$ & 0.9318 \\
\hline & LE $139.4 \pm 20.6$ & LE $144.3 \pm 16.4$ & 0.2880 \\
\hline \multirow{2}{*}{ N-RNFL $(\mu \mathrm{m})$} & RE $84.4 \pm 15.8$ & RE $87.6 \pm 14.7$ & 0.3345 \\
\hline & LE $84.9 \pm 16.1$ & LE $87.4 \pm 15.0$ & 0.6345 \\
\hline \multirow{2}{*}{ T-RNFL $(\mu \mathrm{m})$} & $\mathrm{RE} 83.9 \pm 15.1$ & $\operatorname{RE} 85.6 \pm 13.9$ & 0.4481 \\
\hline & LE $84.2 \pm 14.3$ & LE $87.3 \pm 13.1$ & 0.6153 \\
\hline \multirow{2}{*}{ FAZ-SP $\left(\mu \mathrm{m}^{2}\right)$} & $\operatorname{RE} 405.0 \pm 136.4$ & RE $307.3 \pm 78.6$ & 0.0014 \\
\hline & LE $477.1 \pm 124.4$ & LE $384.0 \pm 88.7$ & 0.0012 \\
\hline \multirow{2}{*}{ FAZ-DP $\left(\mu \mathrm{m}^{2}\right)$} & $\operatorname{RE} 477.1 \pm 124.4$ & RE $384.4 \pm 88.7$ & 0.0012 \\
\hline & LE $497.9 \pm 124.1$ & LE $484.9 \pm 92.7$ & 0.0005 \\
\hline
\end{tabular}




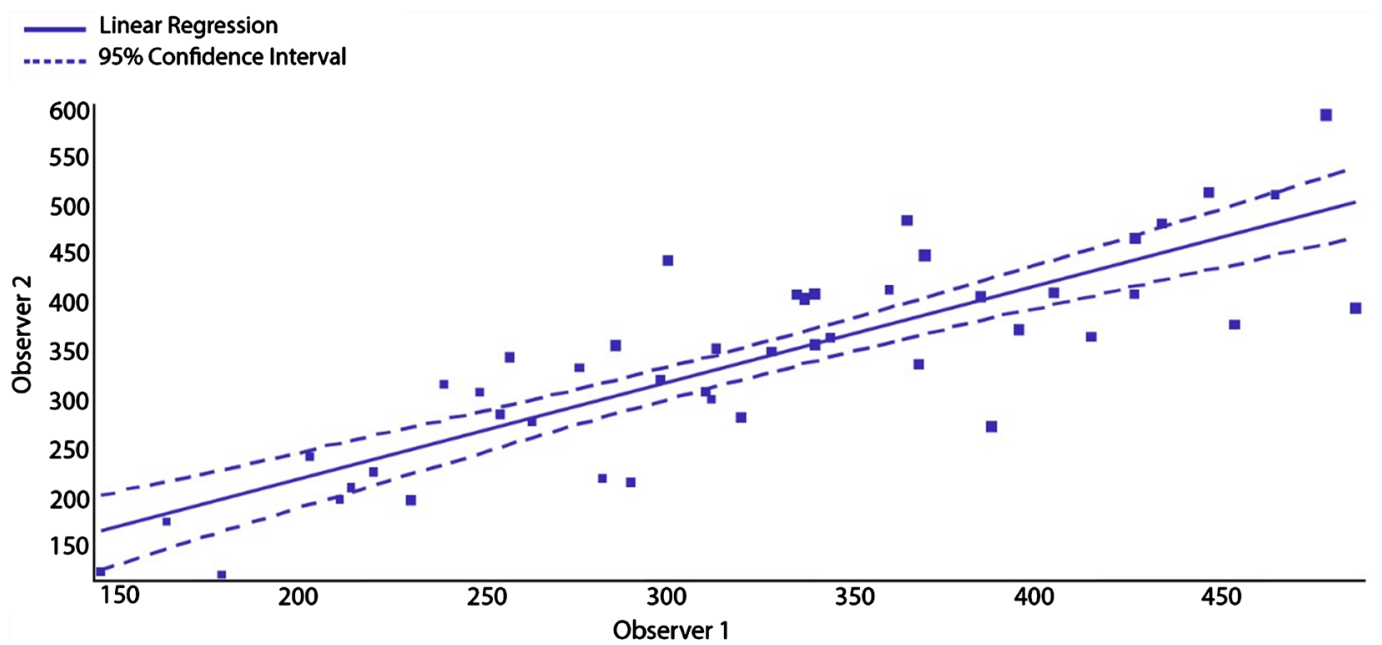

Figure 1. Correlation between FAZ measurements made by independent observers $(r=0.7024)$.

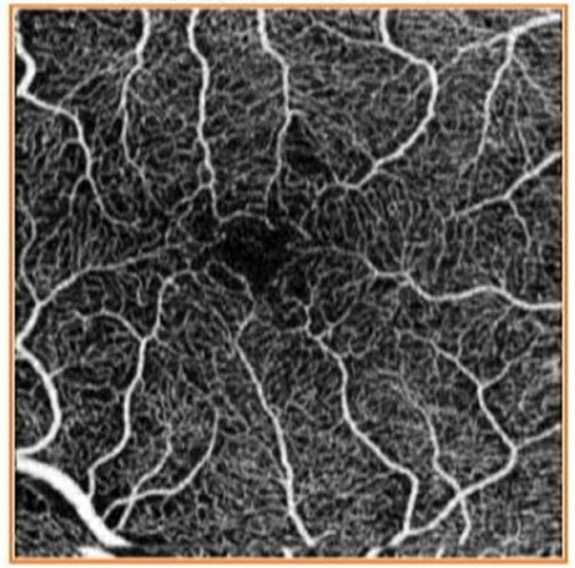

(a)

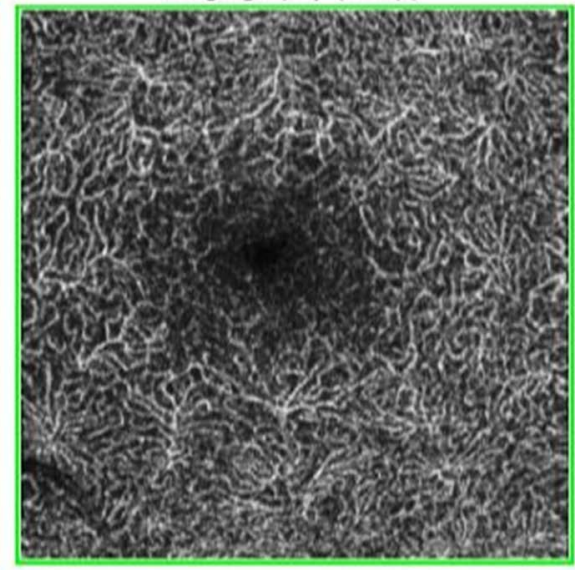

(b)

Figure 2. OCT-A $(3 \times 3 \mathrm{~mm})$ of the right eye of a 40 -year-old control group individual, female: (a) superficial plexus and (b) deep plexus.

In some obese, with and without comorbidities, the widening of the FAZ-SP and FAZ-DP and signs of retinal ischemia were demonstrated in their OCT-A images (Figure 3 and Figure 4). One of the patients in the study group had type 2 diabetes showing signs of ischemia in his OCT-A with enlargement and distortion of the shape of his FAZ-SP and FAZ-DP (Figure 4). This was the only diabetic patient (out of a total of 10 in the study group) who had signs of diabetic retinopathy on OCT-A.

In the study group, the FAZ area in the superficial plexus was significantly smaller than the FAZ area in the deep plexus ( $p=0.0152$ in the right eye and $p=$ 0.0053 in the left eye) and the correlations between the FAZ area in the superficial plexus and the FAZ area in deep plexus were also significant $(\mathrm{p}<0.0001, \mathrm{r}=$ $0.7032,95 \%$ CI $0.4832-0.8397$ in the right eye and $\mathrm{p}<0.0001, \mathrm{r}=0.8874,95 \%$ CI $0.7829-0.9432$ in the left eye) (Figure 5).

In the control group, the area of the FAZ in the superficial plexus was significantly smaller than the area of the FAZ in the deep plexus ( $p=0.0008$ in the 
right eye and $\mathrm{p}=0.0023$ in the left eye) and the correlation between the area of the FAZ in the superficial and deep plexus was also significant $(\mathrm{p}<0.0001, \mathrm{r}=$ $0.8485,95 \%$ CI $0.7211-0.9308$ in the right eye and $\mathrm{p}<0.0001, \mathrm{r}=0.8663,95 \%$ CI $0.7356-0.9348$ in the left eye) (Figure 6).

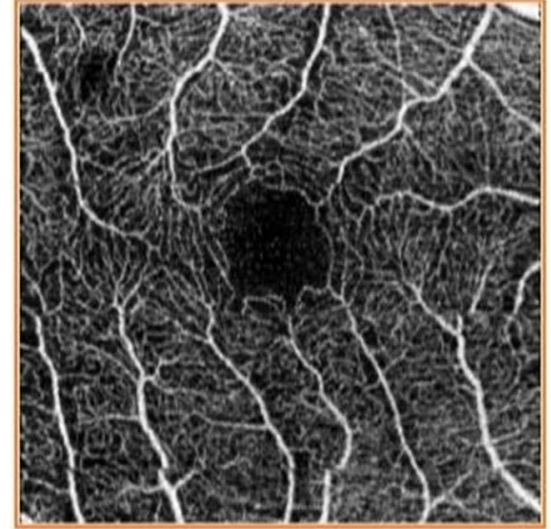

(a)

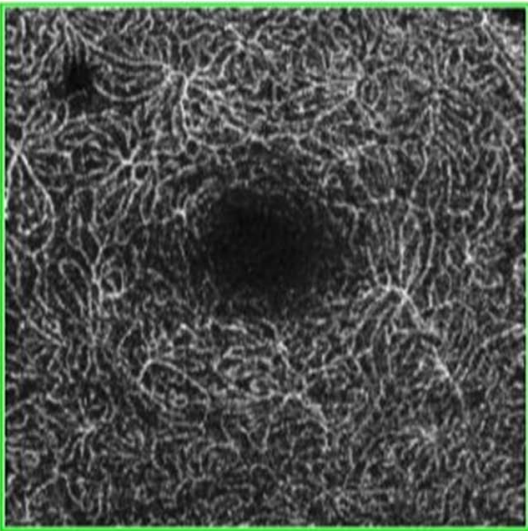

(b)

Figure 3. OCT-A $(3 \times 3 \mathrm{~mm})$ of the right eye of a 46 -year-old female in the study group, without comorbidities: (a) superficial plexus and (b) deep plexus.

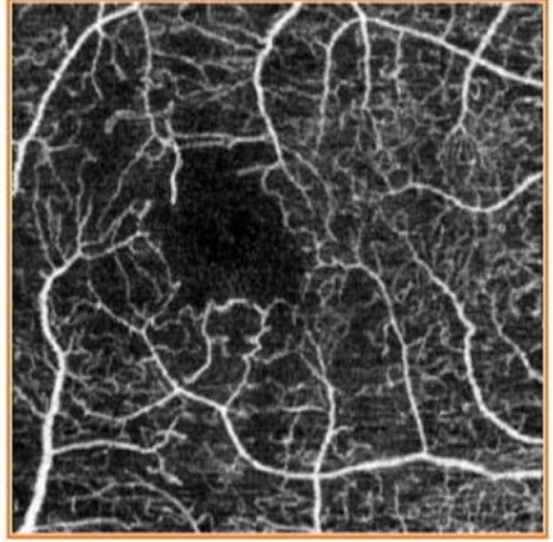

(a)

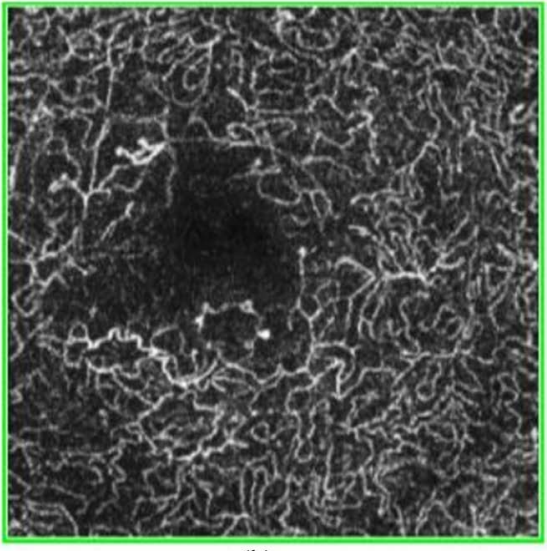

(b)

Figure 4 . OCT-A $(3 \times 3 \mathrm{~mm})$ of the right eye of a study group patient (46 years old, male with type 2 diabetes mellitus): (a) superficial plexus and (b) deep plexus.

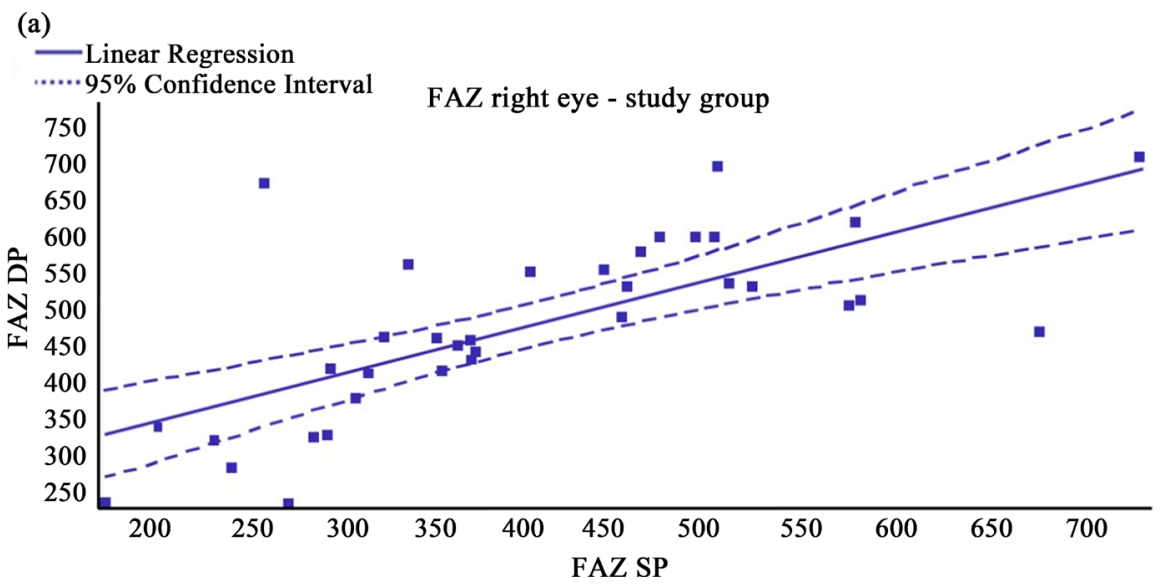




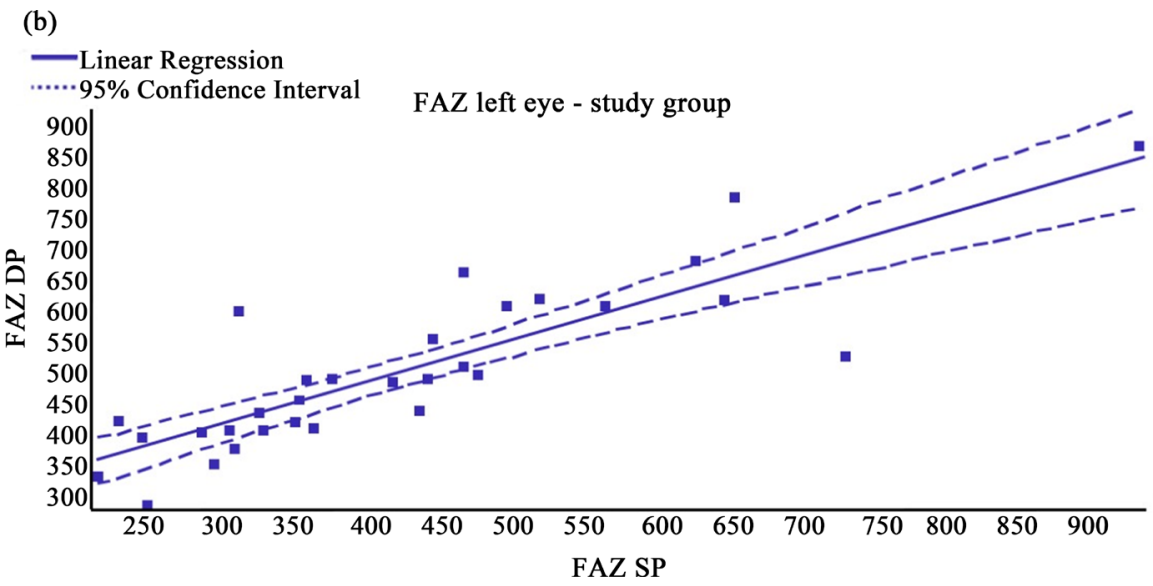

Figure 5. Correlation between FAZ in superficial plexus and FAZ in deep plexus in obese. (a) right eye and (b) left eye.

(a)

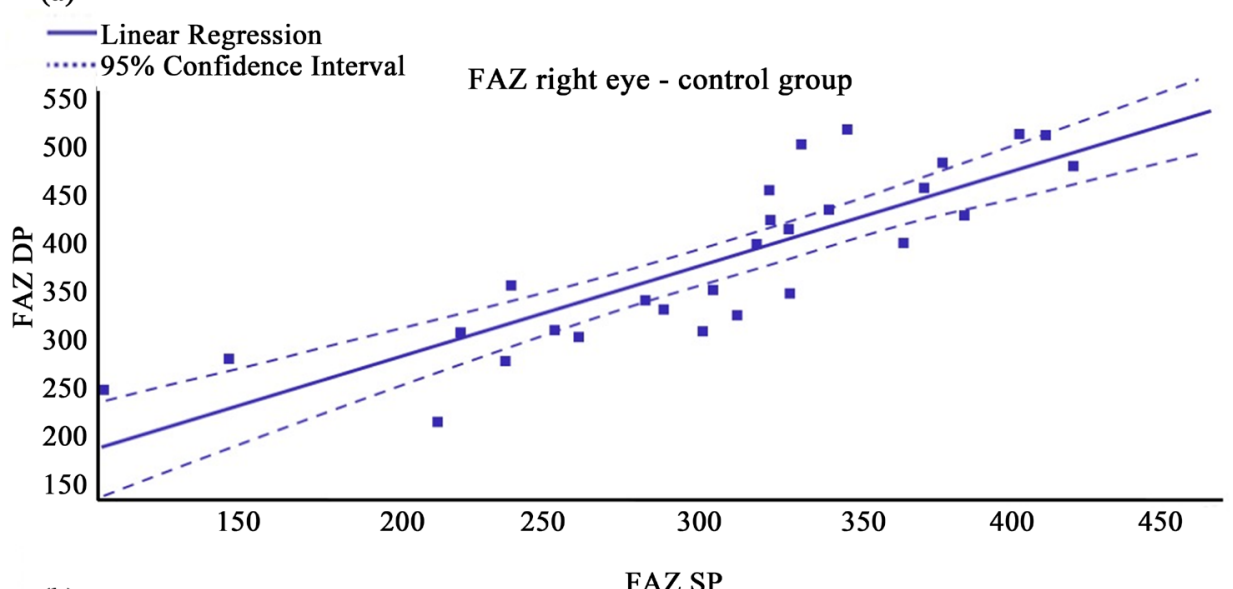

(b)

FAZ SP

Linear Regression
......95\% Confidence Interval

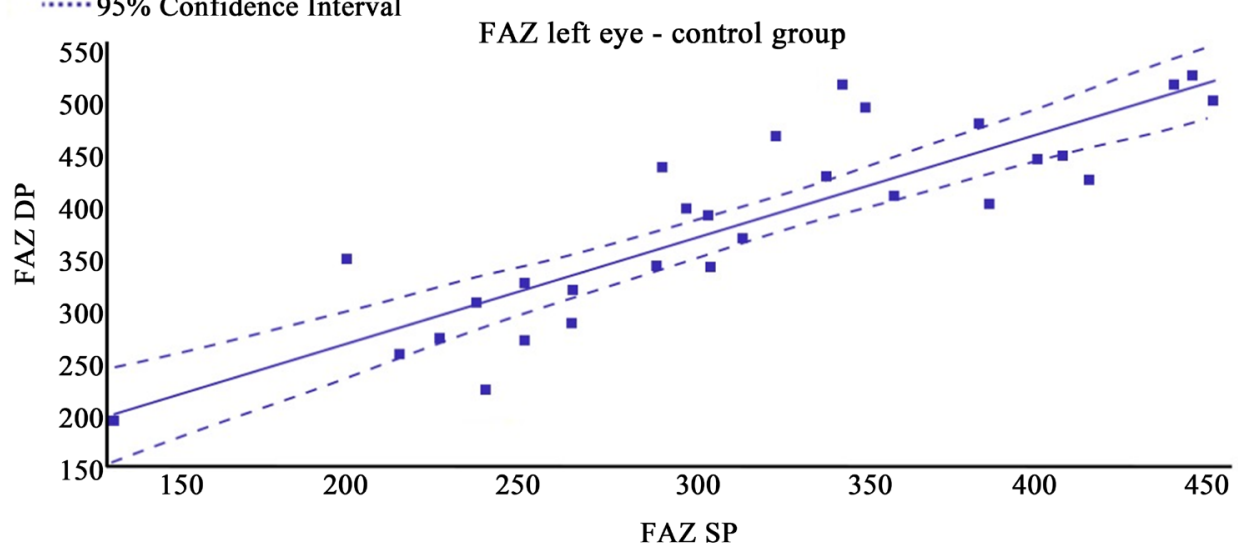

Figure 6. Correlation between FAZ in superficial plexus and FAZ in deep plexus in the control group. (a) right eye and (b) left eye.

There were no correlations with FAZ-SP and FAZ-DP in both eyes with fasting blood glucose, glycated hemoglobin, total cholesterol and fractions and triglycerides (Table 3 to Table 8). 
Table 3. Correlation between laboratory tests and FAZ.

\begin{tabular}{ccc}
\hline \multirow{2}{*}{ Measures } & \multicolumn{2}{c}{ Fasting blood glucose } \\
\cline { 2 - 3 } & r value & p value \\
\hline \multirow{2}{*}{ FAZ-SP $\left(\mu \mathrm{m}^{2}\right)$} & $\mathrm{RE}-0.0752$ & 0.6678 \\
& $\mathrm{LE}-0.1917$ & 0.2699 \\
& $\mathrm{RE} 0.2932$ & 0.0874 \\
FAZ-DP $\left(\mu \mathrm{m}^{2}\right)$ & $\mathrm{LE}-0.0187$ & 0.9149 \\
\hline
\end{tabular}

Table 4. Correlation between laboratory tests and FAZ.

\begin{tabular}{ccc}
\hline \multirow{2}{*}{ Measures } & \multicolumn{2}{c}{ Glycated hemoglobin } \\
\cline { 2 - 3 } & r value & p value \\
\hline \multirow{2}{*}{ FAZ-SP $\left(\mu \mathrm{m}^{2}\right)$} & $\mathrm{RE} 0.0502$ & 0.7747 \\
& $\mathrm{LE}-0.1616$ & 0.3538 \\
& & 0.1585 \\
FAZ-DP $\left(\mu \mathrm{m}^{2}\right)$ & $\mathrm{RE} 0.2436$ & 0.6506 \\
& $\mathrm{LE}-0.0793$ & \\
\hline
\end{tabular}

Table 5. Correlation between laboratory tests and FAZ.

\begin{tabular}{ccc}
\hline \multirow{2}{*}{ Measures } & \multicolumn{2}{c}{ Total cholesterol } \\
\cline { 2 - 3 } & r value & p value \\
\hline \multirow{2}{*}{ FAZ-SP $\left(\mu \mathrm{m}^{2}\right)$} & $\mathrm{RE} 0.0472$ & 0.7874 \\
& $\mathrm{LE}-0.0891$ & 0.6107 \\
FAZ-DP $\left(\mu \mathrm{m}^{2}\right)$ & $\mathrm{RE} 0.0142$ & 0.9356 \\
& $\mathrm{LE}-0.0823$ & 0.6385 \\
\hline
\end{tabular}

Table 6. Correlation between laboratory tests and FAZ.

\begin{tabular}{ccc}
\hline \multirow{2}{*}{ Measures } & \multicolumn{2}{c}{ Triglycerides } \\
\cline { 2 - 3 } & r value & p value \\
\hline \multirow{2}{*}{ FAZ-SP $\left(\mu \mathrm{m}^{2}\right)$} & $\mathrm{RE}-0.054$ & 0.7520 \\
& $\mathrm{LE}-0.2457$ & 0.1548 \\
FAZ-DP $\left(\mu \mathrm{m}^{2}\right)$ & $\mathrm{RE} 0.0373$ & 0.8315 \\
& $\mathrm{LE}-0.1225$ & 0.4833 \\
\hline
\end{tabular}

Table 7. Correlation between laboratory tests and FAZ.

\begin{tabular}{ccc}
\hline \multirow{2}{*}{ Measures } & \multicolumn{2}{c}{ High-density lipoprotein $(\mathrm{HDL})$} \\
\cline { 2 - 3 } & r value & p value \\
\hline FAZ-SP $\left(\mu \mathrm{m}^{2}\right)$ & $\mathrm{RE}-0.1595$ & 0.3599 \\
& LE 0.0245 & 0.8887 \\
FAZ-DP $\left(\mu \mathrm{m}^{2}\right)$ & $\mathrm{RE}-0.1566$ & 0.3691 \\
& LE 0.0048 & 0.9780 \\
\hline
\end{tabular}


Table 8. Correlation between laboratory tests and FAZ.

\begin{tabular}{ccc}
\hline \multirow{2}{*}{ Measures } & \multicolumn{2}{c}{ Low-density lipoprotein (LDL) } \\
\cline { 2 - 3 } & r value & p value \\
\hline \multirow{2}{*}{ FAZ-SP $\left(\mu \mathrm{m}^{2}\right)$} & RE 0.0908 & 0.6036 \\
& LE -0.0980 & 0.5752 \\
FAZ-DP $\left(\mu \mathrm{m}^{2}\right)$ & RE 0.0347 & 0.8432 \\
& LE -0.1126 & 0.5197 \\
\hline
\end{tabular}

No significant association was found between FAZ-SP and comorbidities and life habits. Although, there was a significant association between a larger deep FAZ area and type 2 diabetes mellitus $(\mathrm{p}=0.0160)$.

\section{Discussion}

The choice of an imaging method that assesses the effect of obesity in the retinal microcirculation is a relevant topic, which can help the management of this disease by assessing the hemodynamic status in the vascular plexuses of the retina, including the measurement of the foveal avascular zone. OCT-A comes as a promising option, providing a better study and understanding of ocular involvement in obesity, including the possibility of predicting changes that would only be detected after fundoscopy and fluorescein angiography exam, as has been described for several vascular diseases of the retina [15]-[20].

So far, to the best of our knowledge, the present study was the first to assess, through the OCT-A, FAZ in obese individuals, checking for possible relationships between FAZ and comorbidities, including parameters of clinical evaluation, biochemical tests and lifestyle habits.

Obesity, as a disease of multifactorial etiology and risk factor for several comorbidities, requires a multidisciplinary team approach and the ophthalmologist must be an integral part of this preventive and therapeutic intervention, especially in the control of chronic non-communicable diseases that have ocular repercussions [3]-[11].

In the ophthalmological evaluation, significantly greater values of intraocular pressure were found in obese in both eyes, corroborating a finding already described in other studies in the literature [9] [10]. The increased intraocular pressure (IOP) in obese does not yet have a well-described pathophysiological mechanism, which may be related to hyperleptinemia present in obesity, which can trigger oxidative damage to the trabecular mesh, or else to the increase in orbital fat and blood viscosity, which increase episcleral venous pressure. and reduces the flow of aqueous humor [21].

FAZ has the fluorescein angiography as the gold standard image exam for its evaluation, although without the possibility of differentiating between superficial and deep vasculature of the retina [22]. Thus, assessment of the FAZ has been consolidated through the OCT-A, which allows visualization of the superficial and deep capillary plexuses of the retina allowing the delimitation of the superfi- 
cial and deep foveal avascular zones and calculation of their areas. To determine the relationship between variation in the area of the FAZ and retinal diseases, it is essential to understand the variation of the FAZ in healthy individuals [16] [17] [18] [19] [23].

In obese as well as in the control group, the area of the FAZ in the superficial plexus was significantly smaller than the area of the FAZ in the deep plexus, coinciding with findings already found in other studies [24]. There was a significant correlation between the FAZ area in the superficial plexus and the FAZ area in the deep plexus in both eyes, as described in the literature [25]. The larger area of the FAZ in the deep plexus can be explained in part because in that plexus the vessels are actually moving into the inner plexiform layer/ganglion cell layer as all of these anatomic layers narrow at the fovea [24].

The size of the FAZ reflects the status of the capillaries in the macular area and has a strong correlation with the severity of capillary non-perfusion, which can be observed in retinal diseases of an ischemic nature, with the possibility of decreasing the retina microvasculature and enlarging this area due to vascular interruption [15]-[20].

BMI can result in reduced capillary density of the skin and less tissue perfusion, one of the first detectable changes in microvascular function in overweight individuals [15]. Mechanisms already described associated with high BMI such as changes in hormone levels (increase in leptin levels and decrease in ghrelin levels), vasodilators (decrease in nitric oxide levels), vasoconstrictors (increase in endothelin-1 and angiotensin-II levels), induction of neurotoxicity, decrease in blood flow, mechanical factors and oxidative stress may play an important role in the development of eye diseases [9], including microangiopathy in retinal vessels, with possible change in vascular density and widening of the foveal avascular zone in obese people compared to patients with BMI in the normal range. In the present study, the means of FAZ areas in the superficial and deep plexus achieved significantly greater values in the obese in both eyes.

In relation to comorbidities, in the study group, a significant association was found between increasing of the FAZ area in deep plexus and type 2 diabetes mellitus. Diabetic eyes are known to present impairment of macular microcirculation even before the onset of retinopathy and the assessment of the area of the FAZ has been used for the early detection of diabetic retinopathy [26] [27]. The perifoveal intercapillary area in OCT-A appears to increase in size as the level of diabetic retinopathy progresses [20]. The increased FAZ area in the superficial and deep plexus can detect the lack of integrity of the vascular arches around the FAZ in diabetics, with vascular abnormalities being more pronounced in the deep vascular layer [16].

No relation was found between increasing of the FAZ area in the superficial and deep plexus and systemic arterial hypertension in this study. However, there are reports of changes in the retina microvasculature in hypertensive patients through evaluation by OCT-A [18]. The area of the FAZ was significantly en- 
larged and the vascular density of the retina significantly reduced in hypertensive patients compared to healthy patients, suggesting that OCT-A can identify early hypertensive damage helping to monitor disease progression [18] [19].

In the present study, no significant differences were found between the measurements taken of central macula thickness, subfoveal choroidal thickness, mean peripapillary retinal nerve fiber layer thickness, superior peripapillary retinal nerve fiber layer thickness, inferior peripapillary retinal nerve fiber layer thickness, nasal peripapillary retinal nerve fiber layer thickness and temporal peripapillary retinal nerve fiber layer thickness between the study group and the control group. It is interesting to note that there are studies that describe a decrease in the subfoveal choroidal thickness [9], a decrease in the thickness of the retinal nerve fiber layer [9] [11] and an increase in macular thickness [8] in obese individuals. It is possible that these findings are due to samples with different characteristics such as refractive errors, for example.

The correlations between laboratory tests and measurements of superficial and deep FAZ areas were not significant. As already described, the involvement of macular microcirculation in diabetics [16] [17] [22] could be corroborated by the correlation with laboratory tests such as fasting blood glucose and glycated hemoglobin, however, there was not verified these correlations in the present study. Values related to the lipid profile could have shown a correlation with a larger FAZ area [28], however, such correlations were not found.

In relation to life habits, no association was observed between FAZ areas in superficial and deep plexus with smoking and physical activity, probably due to the sample size, since there are reports in the literature of enlargement of FAZ areas in smokers [29] and smaller FAZ areas in practitioners of physical activity [30].

Study limitations: the main limitations of the present study are the relatively small sample size and the heterogeneity of clinical data, since obese individuals have several comorbidities that affect clinical parameters and which may eventually influence the findings in OCT and OCT-A. It was not possible compare within the obese group diabetes versus non-diabetes because of the small sample size. The larger sample size and a multivariate analysis, which is particularly necessary in the evaluation of patients with multiple comorbidities, could corroborate the association between obesity and changes in imaging tests. Regarding the possible measurement error of independent observer, it was observed that there was no difference between the mean of the measurements of the two observers and a strong correlation was found between these measurements, and good accuracy should be considered in the measurements performed of the FAZ of the obese and the control group recruited for this study.

\section{Conclusion}

The FAZ areas in superficial and deep plexus achieved significantly greater values in the study group. There was a significant association between a larger deep 
FAZ area and type 2 diabetes mellitus. It is necessary an evaluation with a larger sample size to corroborate the findings.

\section{Conflicts of Interest}

The authors declare no conflicts of interest regarding the publication of this paper.

\section{References}

[1] World Health Organization (2020) Obesity and Overweight. World Health Organization, Geneva. https://www.who.int/mediacentre/factsheets/fs311/en/

[2] Blüher, M. (2019) Obesity: Global Epidemiology and Pathogenesis. Nature Reviews Endocrinology, 15, 288-298. https://doi.org/10.1038/s41574-019-0176-8

[3] Boles, A., Kandimalla, R. and Reddy, P.H. (2017) Dynamics of Diabetes and Obesity: Epidemiological Perspective. Biochimica et Biophysica Acta (BBA)-Molecular Basis of Disease, 1863, 1026-1036. https://doi.org/10.1016/j.bbadis.2017.01.016

[4] Piché, M.E., Poirier, P., Lemieux, I. and Després, J.P. (2018) Overview of Epidemiology and Contribution of Obesity and Body Fat Distribution to Cardiovascular Disease: An Update. Progress in Cardiovascular Diseases, 61, 103-113. https://doi.org/10.1016/j.pcad.2018.06.004

[5] Lakkis, J.I. and Weir, M.R. (2018) Obesity and Kidney Disease. Progress in Cardiovascular Diseases, 61, 157-167. https://doi.org/10.1016/j.pcad.2018.07.005

[6] Salaün, H., Thariat, J., Vignot, M., Merrouche, Y. and Vignot, S. (2017) Obesity and Cancer. Bulletin du Cancer, 104, 30-41. https://doi.org/10.1016/j.bulcan.2016.11.012

[7] Zhang, Q.Y., Tie, L.J., Wu, S.S., Lv, P.L., Huang, H.W. and Wang, W.Q. (2016) Overweight, Obesity, and Risk of Age-Related Macular Degeneration. Investigative Ophthalmology \& Visual Science, 57, 1276-1283.

https://doi.org/10.1167/iovs.15-18637

[8] Panon, N., Luangsawang, K., Rugaber, C., Tongchit, T., Thongsepee, N., Cheaha, D., Kongjaidee, P., Changtong, A., Daradas, A. and Chotimol, P. (2019) Correlation between Body Mass Index and Ocular Parameters. Clinical Ophthalmology, 13, 763-769. https://doi.org/10.2147/OPTH.S196622

[9] Dogan, B., Kazim Erol, M., Dogan, U., Habibi, M., Bulbuller, N., Turgut Coban, D. and Bulut, M. (2016) The Retinal Nerve Fiber Layer, Choroidal Thickness, and Central Macular Thickness in Morbid Obesity: An Evaluation Using Spectral-Domain Optical Coherence Tomography. European Review for Medical and Pharmacological Sciences, 20, 886-891.

[10] Teberik, K., Eski, M.T., Doğan, S., Pehlivan, M. and Kaya, M. (2019) Ocular Abnormalities in Morbid Obesity. Arquivos Brasileiros de Oftalmologia, 82, 6-11. https://doi.org/10.5935/0004-2749.20190007

[11] Laiginhas, R., Guimarães, M., Cardoso, P., Santos-Sousa, H., Preto, J., Nora, M., Chibante, J., Falcão-Reis, F. and Falcão, M. (2019) Retinal Nerve Fiber Layer Thickness Decrease in Obesity as a Marker of Neurodegeneration. Obesity Surgery, 29, 2174-2179. https://doi.org/10.1007/s11695-019-03806-7

[12] Nartoli, R., Fernando, N., Dahlenburg, T., Jiao, H., Aggio-Bruce, R., Barnett, N.L., et al. (2018) Obesity-Induced Metabolic Disturbance Drives Oxidative Stress and Complement Activation in the Retinal Environment. Molecular Vision, 24, 201-217.

[13] George, A.K., Singh, M., Homme, R.P., Majumder, A., Sandhu, H.S. and Tyagi, S.C. 
(2018) A Hypothesis for Treating Inflammation and Oxidative Stress with Hydrogen Sulfide during Age-Related Macular Degeneration. International Journal of Ophthalmology, 11, 881-887.

[14] Godoy, E.P., Pereira, S.S.S., Coelho, D.P., Medeiros, I.M., Luz, V.F., Coutinho, J.L., Palitot, T.R.C., Costa, H.B.F., Campos, J.M. and Brandt, C.T. (2019) Bipartição de trânsito intestinal isolada: Uma nova estratégia para cirurgia em estágios em superobesos. Revista do Colégio Brasileiro de Cirurgióes, 46, Article ID: e20192264. https://doi.org/10.1590/0100-6991e-20192264

[15] Czernichow, S., Greenfield, J.R., Galan, P., Bastard, J.P., Charnaux, N., Samaras, K., Safar, M.E., Blacher, J., Hercberg, S. and Levy, B.I. (2010) Microvascular Dysfunction in Healthy Insulin-Sensitive Overweight Individuals. Journal of Hypertension, 28, 325-332. https://doi.org/10.1097/HJH.0b013e328333d1fc

[16] Freiberg, F.J., Pfau, M. and Wons, J. (2016) Optical Coherence Tomography Angiography of the Foveal Avascular Zone in Diabetic Retinopathy. Graefe's Archive for Clinical and Experimental Ophthalmology, 254, 1051-1058.

https://doi.org/10.1007/s00417-015-3148-2

[17] Garcia, J.M., Lima, T.T., Louzada, R.N., Rassi, A.T., Isaac, D.L. and Avila, M. (2016) Diabetic Macular Ischemia Diagnosis: Comparison between Optical Coherence Tomography Angiography and Fluorescein Angiography. Journal of Ophthalmology, 2016, Article ID: 3989310. https://doi.org/10.1155/2016/3989310

[18] Donati, S., Maresca, A.M., Cattaneo, J., Grossi, A., Mazzola, M., Caprani, S.M., Premoli, L., Docchio, F., Rizzoni, D., Gaste, L. and Azzolini, C. (2019) Optical Coherence Tomography Angiography and Arterial Hypertension: A Role in Identifying Subclinical Microvascular Damage? European Journal of Ophthalmology, 31, 158-165. https://doi.org/10.1177\%2F1120672119880390

[19] Takayama, K., Kaneko, H., Ito, Y., Kataoka, K., Iwase, T., Yasuma, T., Matsuura, T., Tsunekawa, T., Shimizu, H., Suzumura, A., Ra, E., Akahori, T. and Terasaki, H. (2018) Novel Classification of Early Stage Systemic Hypertensive Changes in $\mathrm{Hu}$ man Retina Based on OCTA Measurement of Choriocapillaris. Scientific Reports, 8 , Article No. 15163. https://doi.org/10.1038/s41598-018-33580-y

[20] Salz, D.A., de Carlo, T.E. and Adhi, M. (2016) Select Features of Diabetic Retinopathy on Swept-Source Optical Coherence Tomographic Angiography Compared with Fluorescein Angiography and Normal Eyes. JAMA Ophthalmology, 134, 644-650. https://doi.org/10.1001/jamaophthalmol.2016.0600

[21] Stojanov, O., Stokić, E., Sveljo, O. and Naumović, N. (2013) The Influence of Retrobulbar Adipose Tissue Volume upon Intraocular Pressure in Obesity. Vojnosanitetski pregled, 70, 469-476. https://doi.org/10.2298/VSP1305469S

[22] Dmuchowska, D.A., Krasnicki, P. and Mariak, Z. (2014) Can Optical Coherence Tomography Replace Fluorescein Angiography in Detection of Ischemic Diabetic Maculopathy? Graefe's Archive for Clinical and Experimental Ophthalmology, 252, 731-738. https://doi.org/10.1007/s00417-013-2518-x

[23] Fujiwara, A., Morizane, Y. and Hosokawa, M. (2017) Factors Affecting Foveal Avascular Zone in Healthy Eyes: An Examination Using Swept-Source Optical Coherence Tomography Angiography. PLoS ONE, 12, e0188572.

https://doi.org/10.1371/journal.pone.0188572

[24] Coscas, F., Sellam, A. and Glacet-Bernard, A. (2016) Normative Data for Vascular Density in Superficial and Deep Capillary Plexuses of Healthy Adults Assessed by Optical Coherence Tomography Angiography. Investigative Ophthalmology \& Visual Science, 57, OCT211-OCT223. https://doi.org/10.1167/iovs.15-18793 
[25] Gómez-Ulla, F., Cutrin, P., Santos, P., Fernandez, M., Abraldes, M., Abalo-Lojo, J.M. and Gonzalez, F. (2019) Age and Gender Influence on Foveal Avascular Zone in Healthy Eyes. Experimental Eye Research, 189, Article ID: 107856. https://doi.org/10.1016/j.exer.2019.107856

[26] de Carlo, T.E., Chin, A.T., Bonini Filho, M.A., Adhi, M., Branchini, L., Salz, D.A., et al. (2015) Detection of Microvascular Changes in Eyes of Patients with Diabetes but Not Clinical Diabetic Retinopathy Using Optical Coherence Tomography Angiography. Retina, 35, 2364-2370. https://doi.org/10.1097/IAE.0000000000000882

[27] Takase, N., Nozaki, M., Kato, A., Ozeki, H., Yoshida, M. and Ogura, Y. (2015) Enlargement of Foveal Avascular Zone in Diabetic Eyes Evaluated by En Face Optical Coherence Tomography Angiography. Retina, 35, 2377-2383.

https://doi.org/10.1097/IAE.0000000000000849

[28] Stefanutti, C., Mesce, D., Pacella, F., Di Giacomo, S., Turchetti, P., Forastiere, M., et al. (2019) Optical Coherence Tomography of Retinal and Choroidal Layers in Patients with Familial Hypercholesterolaemia Treated with Lipoprotein Apheresis. Atherosclerosis Supplements, 40, 49-54.

https://doi.org/10.1016/j.atherosclerosissup.2019.08.031

[29] Çiloğlu, E., Unal, F., Sukgen, E.A., Kocluk, Y. and Dogan, N.C. (2020) Evaluation of Foveal Avascular Zone and Capillary Plexus in Smokers Using Optical Coherence Tomography Angiography. Journal of Current Ophthalmology, 32, 53-57. https://doi.org/10.1016/j.joco.2019.09.002

[30] Alten, F., Nelis, P., Schmitz, B., Brand, S.M. and Eter, N. (2019) Optical Coherence Tomography Angiography as a Future Diagnostic Tool in Sports Medicine? Der Ophthalmologe, 116, 722-727. https://doi.org/10.1007/s00347-019-0897-z 